\title{
Direct comparison between confocal and multiphoton microscopy for rapid histopathological evaluation of unfixed human breast tissue
}

\author{
Tadayuki Yoshitake \\ Michael G. Giacomelli \\ Lucas C. Cahill \\ Daniel B. Schmolze \\ Hilde Vardeh \\ Beverly E. Faulkner-Jones \\ James L. Connolly \\ James G. Fujimoto
}




\title{
Direct comparison between confocal and multiphoton microscopy for rapid histopathological evaluation of unfixed human breast tissue
}

\author{
Tadayuki Yoshitake, ${ }^{a}$ Michael G. Giacomelli, ${ }^{a}$ Lucas C. Cahill, ${ }^{a}$ Daniel B. Schmolze, ${ }^{\text {b, }}$ Hilde Vardeh, \\ Beverly E. Faulkner-Jones, ${ }^{b}$ James L. Connolly, ${ }^{b}$ and James G. Fujimoto ${ }^{a, *}$ \\ ${ }^{a}$ Massachusetts Institute of Technology, Department of Electrical Engineering and Computer Science and Research Laboratory of Electronics, 32 \\ Vassar Street, Cambridge, Massachusetts 02139, United States \\ bHarvard Medical School, Department of Pathology, Beth Israel Deaconess Medical Center, 330 Brookline Avenue, Boston, Massachusetts 02215, \\ United States
}

\begin{abstract}
Rapid histopathological examination of surgical specimen margins using fluorescence microscopy during breast conservation therapy has the potential to reduce the rate of positive margins on postoperative histopathology and the need for repeat surgeries. To assess the suitability of imaging modalities, we perform a direct comparison between confocal fluorescence microscopy and multiphoton microscopy for imaging unfixed tissue and compare to paraffin-embedded histology. An imaging protocol including dual channel detection of two contrast agents to implement virtual hematoxylin and eosin images is introduced that provides high quality imaging under both one and two photon excitation. Corresponding images of unfixed human breast tissue show that both confocal and multiphoton microscopy can reproduce the appearance of conventional histology without the need for physical sectioning. We further compare normal breast tissue and invasive cancer specimens imaged at multiple magnifications, and assess the effects of photobleaching for both modalities using the staining protocol. The results demonstrate that confocal fluorescence microscopy is a promising and cost-effective alternative to multiphoton microscopy for rapid histopathological evaluation of ex vivo breast tissue. @ 2016 Society of Photo-Optical Instrumentation Engineers (SPIE) [DOI: 10.1117/1.JBO.21.12.126021]
\end{abstract}

Keywords: confocal fluorescence microscopy; multiphoton microscopy; breast cancer; optical pathology.

Paper 160705R received Oct. 12, 2016; accepted for publication Dec. 5, 2016; published online Dec. 29, 2016.

\section{Introduction}

Histopathological assessment of stained thinly sectioned tissue specimens under bright-field microscopy is the gold standard for the diagnosis of most neoplasms, including breast cancer. In conventional histopathological protocols, specimens are processed by fixation, dehydration, paraffin embedding, sectioning into thin slices, and staining, which typically delays evaluation of pathology by at least 1 day. Techniques that enable real-time tissue evaluation are of interest for procedures that are highly time sensitive, such as intraoperative assessment of surgical margins.

Breast conservation therapy (BCT), which includes lumpectomy to remove cancerous tissue along with a margin of surrounding healthy tissue, followed by radiation and/or chemotherapy, is a standard of care for early-stage breast cancer and ductal carcinoma in situ (DCIS). To verify complete surgical resection of the cancer, the surgical margins of the lumpectomy are assessed by postoperative histopathological examination. If postoperative histology finds cancer close to, or on, the surgical margins of excised tissue specimens, reexcision is usually recommended. The rate of second surgeries in BCT due to inadequate resection is up to $40 \%$, resulting in an increased financial burden from repeated surgeries, and

*Address all correspondence to: James G. Fujimoto, E-mail: jgfuji@mit.edu

'Present address: City of Hope National Medical Center, Department of Pathology, Duarte, California 91010, United States worsened morbidity and cosmetic outcomes. ${ }^{1-3}$ Therefore methods for performing intraoperative assessment of breast pathology are needed.

One established means of performing intraoperative histology to reduce re-excision rates is frozen section analysis (FSA). In FSA, specimens are frozen, sectioned into thin slices, stained, and examined under bright-field microscopy within 15 to 30 min of excision. ${ }^{4,5}$ However, the freezing process deforms the tissue structure, limits how thin the tissue can be sectioned, and is challenging to perform on fatty tissues, such as breast, that do not freeze well. ${ }^{6,7}$ Furthermore, sensitivity is controversial, with values reported between $65 \%$ and $91 \%, 8,9$ with small tumors and DCIS being the most difficult to assess. ${ }^{10}$ Consequently, FSA for BCT is not utilized at a majority of clinical centers. ${ }^{11}$

An alternative to time-consuming embedding and physical sectioning of fixed tissue is optical sectioning of unfixed tissue. Imaging unfixed tissue specimens has been demonstrated using techniques such as reflectance confocal microscopy (RCM), ${ }^{12-15}$ confocal fluorescence microscopy (CFM) ${ }^{16-21}$ multiphoton microscopy (MPM), ${ }^{22-24}$ structured illumination microscopy, ${ }^{25}$ spectrally encoded confocal microscopy, ${ }^{26}$ and optical coherence tomography. ${ }^{27,28}$ Furthermore, recently it has been demonstrated that fluorescent contrast agents can be rapidly diffused through viable human tissue, enabling fluorescent labeling without fixation. ${ }^{17,24,25}$ 
MPM and CFM enable strong rejection of out of focus light, high axial resolution imaging, rapid imaging speed, and compatibility with fluorescent contrast agents. Presently, only MPM has been comprehensively evaluated for breast pathology assessment. ${ }^{24}$ This study performed a blinded reading of MPM mosaic images color remapped for an H\&E-like appearance from 179 unfixed, discarded, and randomly selected breast tissue specimens from 50 patients. Specimens were mosaic imaged over large fields, fixed, and then corresponding paraffin-embedded H\&E slides were obtained. A blinded reading showed a $95.4 \%$ sensitivity and $93.3 \%$ specificity for identifying invasive cancer and DCIS versus benign breast tissue compared to paraffin-embedded H\&E histology of the same specimens. The high sensitivity and specificity obtained in this study were in part due to the use of exogenous fluorescent stains to generate nuclear contrast, the high resolution of MPM, and remapping fluorescent and second harmonic generation signals to resemble $\mathrm{H} \& \mathrm{E}$ histopathology.

Unfortunately, while MPM has demonstrated high diagnostic agreement with paraffin-embedded histology for breast surgical specimens, compared to CFM, it is complex and extremely costly because of the need for femtosecond lasers. This high cost may be a barrier to clinical acceptance. Alternatively, extensive literature supports the efficacy of RCM for histological evaluation of skin cancer in vivo ${ }^{12,13}$ and for intraoperative assessment. ${ }^{14}$ Further work has demonstrated that fluorescent labels combined with CFM greatly improve diagnostic accuracy for skin lesions. ${ }^{17-21}$ In spite of the extensive work investigating CFM for skin pathology and MPM for breast pathology, studies of CFM for breast pathology have been limited to qualitative evaluations $^{29}$ or comparisons of selected, high magnification image fields ${ }^{30}$ that are difficult to generalize to readings of intact surgical specimens. Furthermore, to date, protocols for H\&Elike rendering of dual channel CFM for breast surgical specimens have not been demonstrated, further complicating comparison to MPM using virtual H\&E (VH\&E) rendering and sources of contrast such as second harmonic generation that are unavailable to CFM. Consequently, it remains unclear if lower cost CFM can perform equivalently to MPM for evaluation of breast surgical specimens using H\&E-like color rendering applied to large surgical specimens.

In this study, we present a direct comparison of CFM, MPM, and paraffin-embedded H\&E histopathology using freshly excised discarded human breast surgical specimens. We demonstrate a new staining protocol using dual contrast agents that can be rapidly applied to tissue specimens for both MPM and CFM imaging and that enable accurate $\mathrm{VH} \& \mathrm{E}$ rendering of histopathology. VH\&E images were generated by using the virtual transillumination microscopy (VTM) algorithm, which enables physically realistic modeling of virtual transillumination white light microscopy images using epi-fluorescence measurements. ${ }^{31}$ The image quality of both CFM and MPM are compared at 20× magnification, for high-resolution evaluation of cellular features, and $10 \times$ magnification, for examination of architectural morphology of specimens. Photobleaching effects are assessed for the compatibility of the staining protocol with higher speed imaging. Degradation of signal-to-background ratio with imaging depth is also compared between CFM and MPM. These results indicate that CFM can be used to produce high quality VH\&E images of unfixed breast tissue with performance similar to MPM when imaging near the tissue surface.

\section{Methods}

\subsection{Specimen Preparation}

Unfixed breast tissues were acquired under protocols approved by the Massachusetts Institute of Technology Committee on the Use of Humans as Experimental Subjects (COUHES) and the Beth Israel Deaconess Medical Center (BIDMC) Committee on Clinical Investigations (CCI). Only discarded and deidentified human tissue specimens that were not required for clinical diagnosis were acquired, and therefore, the protocol was exempt from informed consent. The discarded tissue specimens were kept in chilled Roswell Park Memorial Institute (RPMI) medium to maintain hydration and imaged within $8 \mathrm{~h}$ of excision. Before imaging, excess fat was removed and representative regions (3 to $5 \mathrm{~mm}$ width) of stroma or pathology were exposed. Propidium iodide (PI) and eosin yellow (EY) were chosen as the nuclear and stromal stains, respectively, because of their excellent penetration into unfixed tissue, low cost, rapid binding, and separated emission spectra. Dissected tissue specimens were stained for $2 \mathrm{~min}$ in a solution of distilled water, $40 \mu \mathrm{g} / \mathrm{ml}$ PI and $200 \mu \mathrm{g} / \mathrm{ml}$ EY. The stained specimens were rinsed in buffered saline and placed on a \#1.5 glass coverslip attached to a specimen holder. The specimen holder was filled with saline-soaked biopsy foam pads so that the surface of the tissue specimens maintained contact with the glass coverslip without dehydration. This method enables repeated imaging of the same specimen for several hours without noticeable alteration of cellular structure or fluorescent signal.

\subsection{Confocal Microscope}

A commercial, inverted confocal laser scanning microscope (Zeiss LSM510) was used for CFM imaging. Figure 1(a) shows the schematic of the confocal microscope. A diode laser $(405 \mathrm{~nm})$ simultaneously excites both contrast agents with $0.5-\mathrm{mW}$ power incident on the sample. The laser was introduced into the optical path using a multiband dichroic beam splitter (Zeiss HFT 405/514/633 nm). The LSM510 uses dual galvanometer scanners with a dwell time of $3 \mu$ s per pixel. Fluorescent signals from PI and EY were detected by dual photomultipliers (R6357; Hamamatsu, Inc.) using a dichroic beam splitter (cutoff of $565 \mathrm{~nm}$ ). An additional long-pass filter at $650 \mathrm{~nm}(\mathrm{PI})$ was used to reduce spectral crosstalk to a negligible level. In the EY channel, a long-pass filter at $505 \mathrm{~nm}$ was used to reject autofluorescence and scattered excitation light. The combination of beam splitters and filters above produced a detection range for EY from 525 to $565 \mathrm{~nm}$, and for PI from $650 \mathrm{~nm}$ to the PMT responsivity cutoff near $800 \mathrm{~nm}$. A Plan-APOCHROMAT 0.8 NA $20 \times$ dry objective (Zeiss) and a Plan-NEOFLUAR 0.3 NA $10 \times$ dry objective (Zeiss) were chosen as representative objectives for CFM. The use of a dry objective slightly degrades resolution when imaging through thick tissue, but greatly simplifies imaging in an inverted microscope geometry, which may be advantageous for intraoperative scenarios. Each image frame was acquired at $1024 \times 1024$ pixels. Due to software limitations, the frame time in mosaic mode was significantly longer than the sum of the pixel dwell times, at about $6 \mathrm{~s}$ per frame, or $24 \mathrm{~s}$ with fourfold averaging. With the $20 \times$ and $10 \times$ objectives, the fields of view were 0.45 and $0.9 \mathrm{~mm}$, with pixel sizes of 0.44 and $0.88 \mu \mathrm{m}$ square, respectively. Therefore, $20 \times$ and $10 \times$ CFM mosaic images were generated at a rate of 2 and $0.5 \mathrm{~min} / \mathrm{mm}^{2}$, 


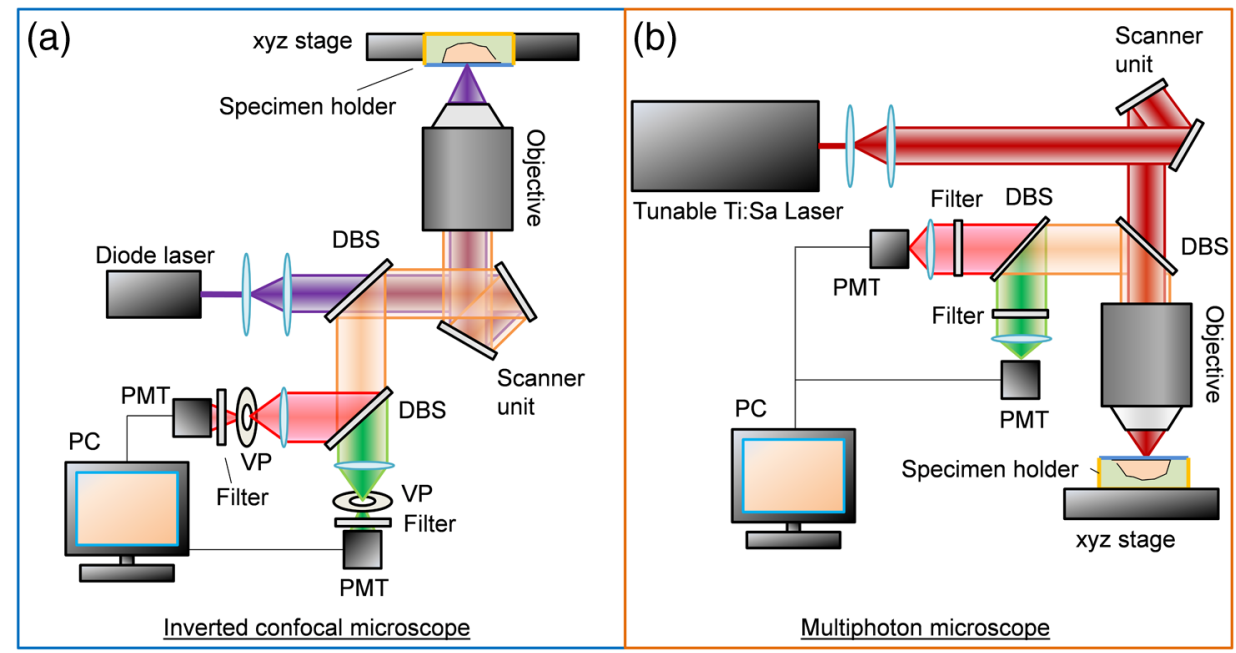

Fig. 1 Schematic of (a) the confocal fluorescence microscope system and (b) the multiphoton microscope system. Both systems used dual channel detectors for nuclear and stromal contrasts. The specimen holder was designed for both inverted and conventional microscope configurations without touching the tissue so that registration could be maintained. (DBS, dichroic beam splitter; VP, variable pinhole; PMT, photomultiplier tube).

respectively. The detector pinhole sizes were set to 1 airy unit for each objective at the detection wavelength range.

\subsection{Multiphoton Microscope}

A commercial nonlinear microscope (Thorlabs, Inc.) was used with a $\sim 150$-fs tunable Ti:sapphire laser (Mira Optima 900-F; Coherent) at $\sim 780 \mathrm{~nm}$ with a $76-\mathrm{MHz}$ repetition rate. Figure 1(b) shows the multiphoton microscope schematic. The excitation laser was scanned by a nonresonant galvanometer scanner and an $8 \mathrm{kHz}(16 \mathrm{kHz}$ bidirectional) resonant galvanometer scanner. The laser power incident on the sample was $<30 \mathrm{~mW}$. Because the fast scan axis moves resonantly, the dwell time per pixel is not uniform along the fast scan axis. The average dwell time per pixel was $60 \mathrm{~ns}$, and minimum dwell time per pixel at the center of the scan line was $40 \mathrm{~ns}$. PI and EY were simultaneously excited, and the fluorescent signals were detected by dual photomultipliers (H7422; Hamamatsu, Inc.) using a dichroic beam splitter (cutoff of $590 \mathrm{~nm}$ ). Additional band-pass filters from 520 to $560 \mathrm{~nm}$ (EY) and from 620 to $680 \mathrm{~nm}$ (PI) were used to reduce spectral crosstalk. A XLUMPFL20XW 1.0 NA 20× water immersion objective (Olympus) and a Plan Apo Lambda 0.45 NA 10× dry objective (Nikon) were used. A water immersion objective was selected for $20 \times$ imaging in order to be consistent with our previous study. ${ }^{24}$ Each image frame was acquired at $1024 \times 1024$ pixels. With the $20 \times$ and $10 \times$ objectives, the fields of view were 0.49 and $1.1 \mathrm{~mm}$, with pixel sizes of 0.48 and $1.05 \mu \mathrm{m}$, respectively. The MPM system operated at 16 frames per second with realtime VH\&E rendering using VTM; however, due to software limitations, $\sim 2 \mathrm{~s}$ were required for mosaic frame acquisition and images were acquired with $50 \%$ overlap, for an imaging rate at $20 \times$ and $10 \times$ magnification of 32 and $8 \mathrm{~s} / \mathrm{mm}^{2}$, respectively. This delay is not present when imaging in live panning mode with VTM.

\subsection{Lateral and Axial Resolutions}

In both CFM and MPM systems, images are laterally undersampled compared with the optical resolution. Therefore, the lateral image resolutions are determined by the sampling intervals which were nearly equal. Conversely, the axial sectioning of each modality is independent of sampling density. In order to characterize the axial resolution of each system, the axial edge was measured using a dilute, homogeneous "fluorescent sea" of fluorescein underneath a coverslip (Table 1) ${ }^{32}$ using the EY channel for detection. The experimentally measured $80 \%$ to $20 \%$ responses at $20 \times$ magnification for both CFM and MPM are comparable to the typical thickness of H\&E histology slides ( $\sim 5 \mathrm{~m}$ ). The $10 \times$ MPM system has about $2 \times$ larger optical sectioning thickness as compared with H\&E histology slides, while the $10 \times$ CFM has about $4 \times$ larger optical sectioning thickness than H\&E histology slides. The imaging results and qualitative comparison between $H \& E$ histology slides are presented in the results and discussion sections.

\subsection{Imaging Procedure}

CFM and MPM imaging were conducted within $1 \mathrm{~h}$ of one another, with no additional preparation done between imaging, to minimize any changes in the unfixed samples. Neither the CFM imaging nor the MPM imaging produced a noticeable effect on the subsequent imaging of the other modality. Single plane, en face images were acquired by translating the specimen with xyz stages and capturing frames at multiple positions. Sequential images from the surface of each specimen (about $5 \mu \mathrm{m}$ into the specimen from the glass coverslip surface) and at increasing depth into the specimen $(25,50$, and $75 \mu \mathrm{m}$ from the surface of specimens) were acquired. Because of the low excitation energy per pixel required when imaging highly

Table 1 Experimental measurement of axial resolution. Axial step response was measured by using infinite sea method.

\begin{tabular}{lcccc} 
& $20 \times$ CFM & $20 \times$ MPM & $10 \times$ CFM & $10 \times$ MPM \\
\hline $\begin{array}{l}\text { Axial resolution } \\
(20 \% \text { to } 80 \%\end{array}$ & $5.5 \mu \mathrm{m}$ & $4 \mu \mathrm{m}$ & $19 \mu \mathrm{m}$ & $8.5 \mu \mathrm{m}$ \\
response $)$ & & & & \\
\hline
\end{tabular}



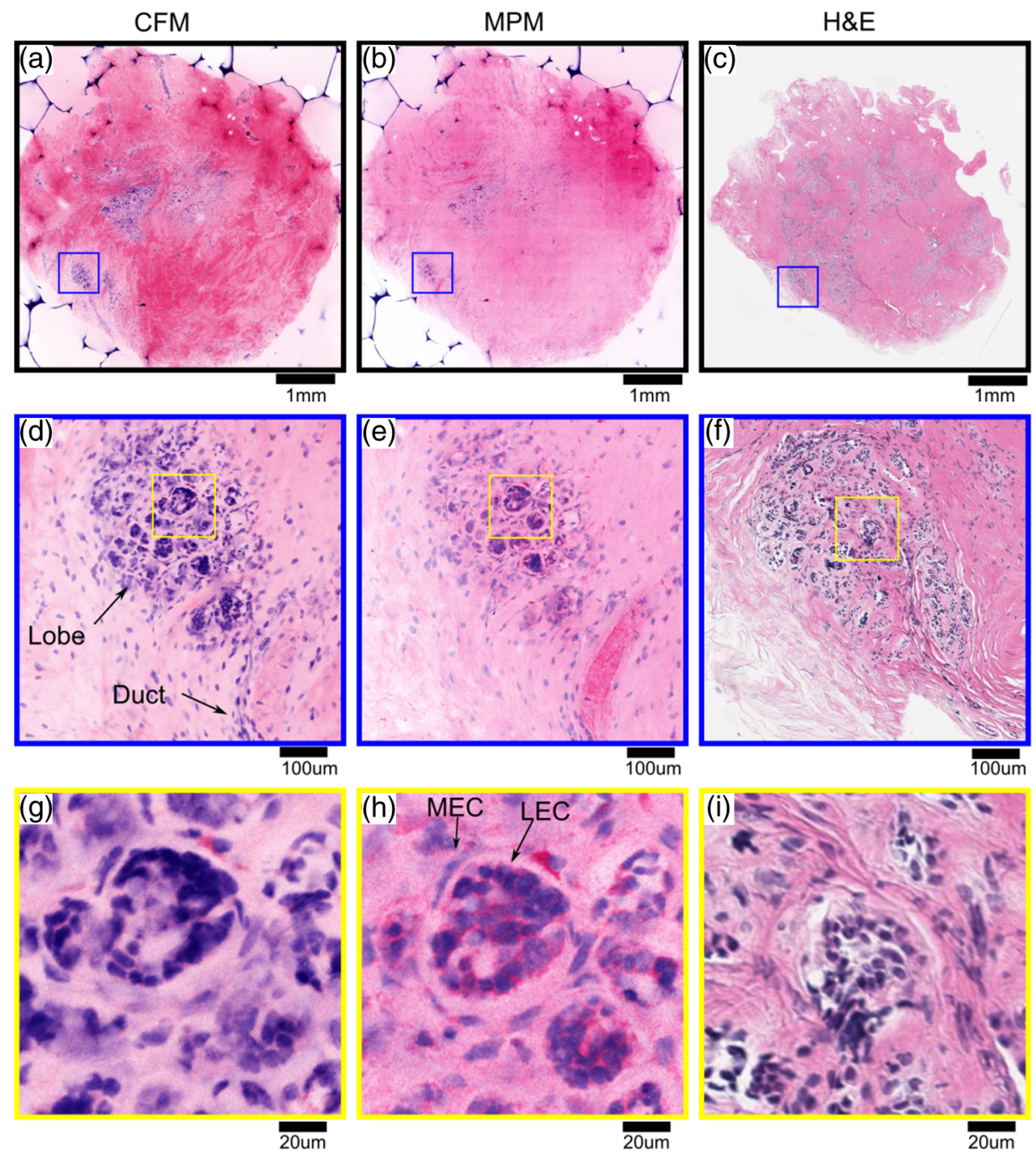

Fig. 2 Comparison of images of normal breast tissue between CFM (20x), MPM (20x), and H\&E histology. (a) CFM image of unfixed breast specimen with TDLUs, (b) corresponding MPM image, and (c) corresponding H\&E histology image showing equivalent low magnification view of the specimen. (d) Magnified CFM image of lobules and a terminal duct, (e) a corresponding MPM image, and (f) corresponding H\&E histology. (g) Higher magnification CFM image of several lobules, (h) a corresponding MPM image, and (i) corresponding H\&E histology all show cellular level structures, including LECs and surrounding MECs.

fluorescent labels and the long collection wavelengths, no autofluorescence was detected from unlabeled tissue on either system. The effect of photobleaching was assessed by examining the signal intensity change from consecutively acquired frames using the 20x objective at the same position on the specimen with distinct regions used for MPM and CFM. After imaging with both CFM and MPM, specimens were fixed using buffered formalin $(10 \%)$ while still immobilized against the specimen holder coverslip in order to retain the image plane orientation. After a minimum of 2 days fixation, the tissue specimens were processed using the standard $\mathrm{H} \& \mathrm{E}$ histology procedure taking care to maintain the specimen orientation during embedding and sectioning and then digitally scanned (Aperio AT2 slide scanner, $20 \times$ magnification; Leica Biosystems $\mathrm{GmbH}$ ). This procedure enables straightforward registration of MPM, CFM, and histology images by minimizing distortion of the tissue during processing.

\subsection{Image Processing}

VH\&E rendering was performed using the VTM algorithm. ${ }^{31}$ VTM is a physically realistic light absorption model that enables high quality rendering of absorption stains such as H\&E using fluorescent data by simulating the transmission of white light through virtual slides, computing a per-dye optical attenuation, and then displaying the remaining transmitted light. The use of a physically realistic model for light absorption enables rendering $\mathrm{H} \& \mathrm{E}$-like images with high dynamic range and more accurate colors than simple color remapping. ${ }^{31}$ Histogram brightness normalization was used to equalize the brightness of both fluorescent channels such that $0.01 \%$ of pixels were overexposed. While VTM enables real-time, live histological evaluation of specimens, wide area mosaics were generated by stitching rendered VH\&E frames using Image Composite Editor (Microsoft Research) in order to compare to histology slides. 

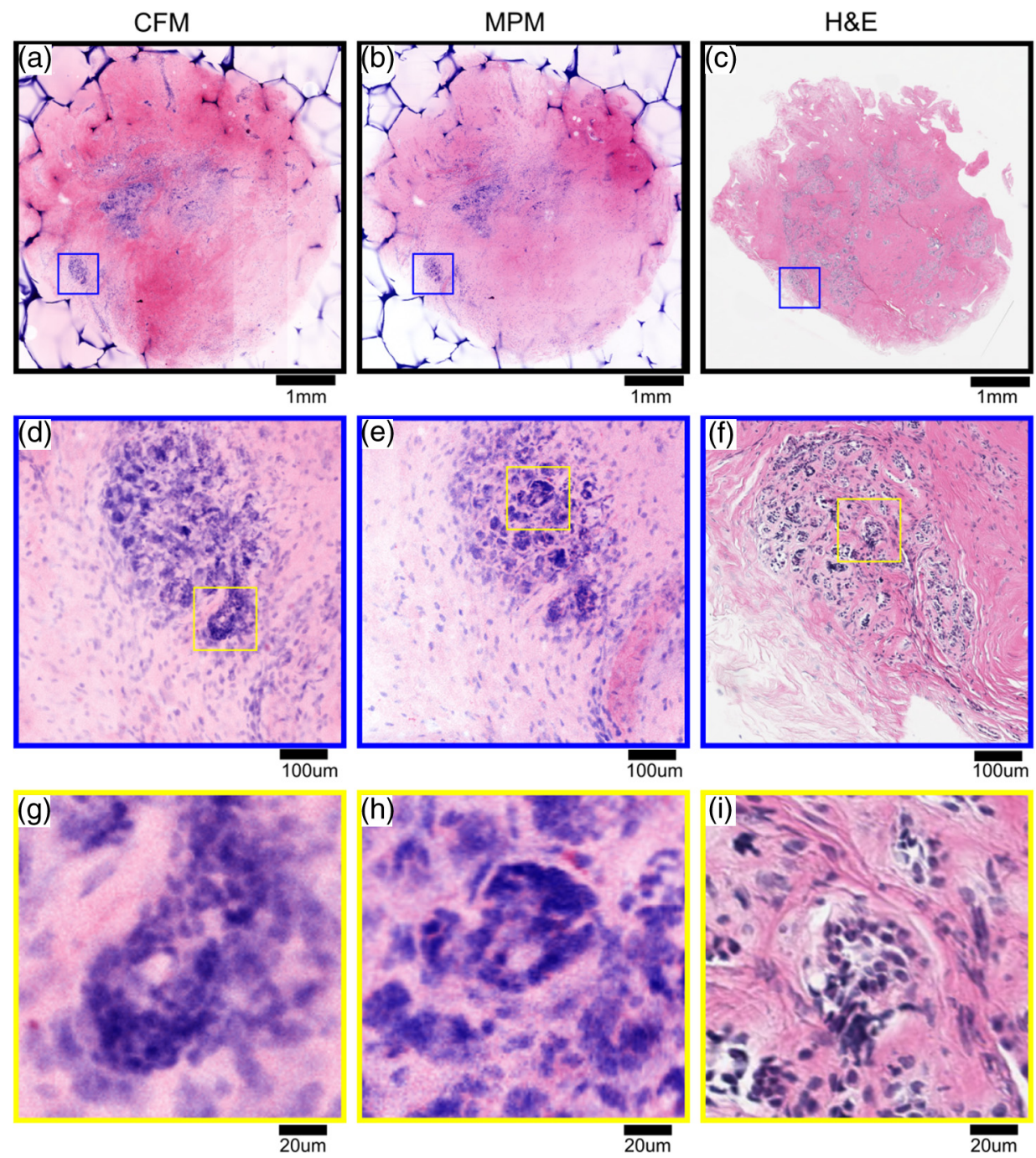

Fig. 3 Comparison of images of normal breast tissue between CFM (10x), MPM (10x), and H\&E histology. (a) CFM image of unfixed breast specimen showing TDLUs, (b) corresponding MPM image, and (c) corresponding H\&E histology image. (d) Magnified CFM image showing a single TDLU, (e) a corresponding MPM image, and (f) magnified view of H\&E histology. (g, h) Higher magnification images of CFM and MPM show blurring, but cellular structures are still identifiable. (i) Further magnified view of H\&E histology. The histology images are identical with those in Fig. 2.

\section{Results}

\subsection{Imaging Results (Surface)}

The surfaces of unfixed breast tissue specimens were evaluated using CFM and MPM, and compared with standard formalin fixed, paraffin-embedded H\&E histology. The surface was defined as the plane $\sim 5 \mu \mathrm{m}$ into the specimen from the glass coverslip surface. CFM and MPM images were obtained using both the $20 \times$ and $10 \times$ objectives. Four frame averaging was conducted for the image acquisition in both CFM and MPM. For purposes of comparing imaging modalities to H\&E histology, we present wide-area mosaic images performed sequentially at each magnification, however, for actual clinical imaging, live panning imaging may be preferred. In breast surgical

margin assessment, specimens are transected (breadloafed) and only the margin of the specimen rather than the entire specimen face needs to be imaged, making mosaic images of the entire specimen inefficient. Example images of normal breast tissue specimens are shown in Figs. 2 and 3, and images of invasive breast cancer specimens are shown in Figs. 4 and 5 .

Figure 2 shows $20 \times$ magnification stitched images of normal breast tissue $(5 \mathrm{~mm} \times 5 \mathrm{~mm})$ from CFM, MPM, and H\&E histology. CFM, MPM, and H\&E histology all show normal terminal duct lobular units (TDLUs) at low magnification (Fig. 2). Magnified images of a 500- $\mu \mathrm{m}$ wide area show a single normal TDLU (Fig. 2, blue box). Higher magnification images of a $100-\mu \mathrm{m}$ wide area demonstrate cellular level constituents, including luminal epithelial cells (LECs) and surrounding myoepithelial cells (MECs), with good concordance between the three modalities. The H\&E histology images [Figs. 2(c), 3(c), 4(c), and 5(c)] show that the specimen deformed and shrank by $\sim 10 \%$ during formalin fixation and paraffin embedding.

Figure 3 shows $10 \times$ images of normal breast tissue $(5 \mathrm{~mm} \times 5 \mathrm{~mm})$ from CFM, MPM, and H\&E histology. Magnified images show the same area size (blue: $500 \mu \mathrm{m}$ wide, black: $100 \mu \mathrm{m}$ wide) as magnified images in Fig. 2. Similar to the $20 \times$ images (Fig. 2), normal TDLUs are seen in the 

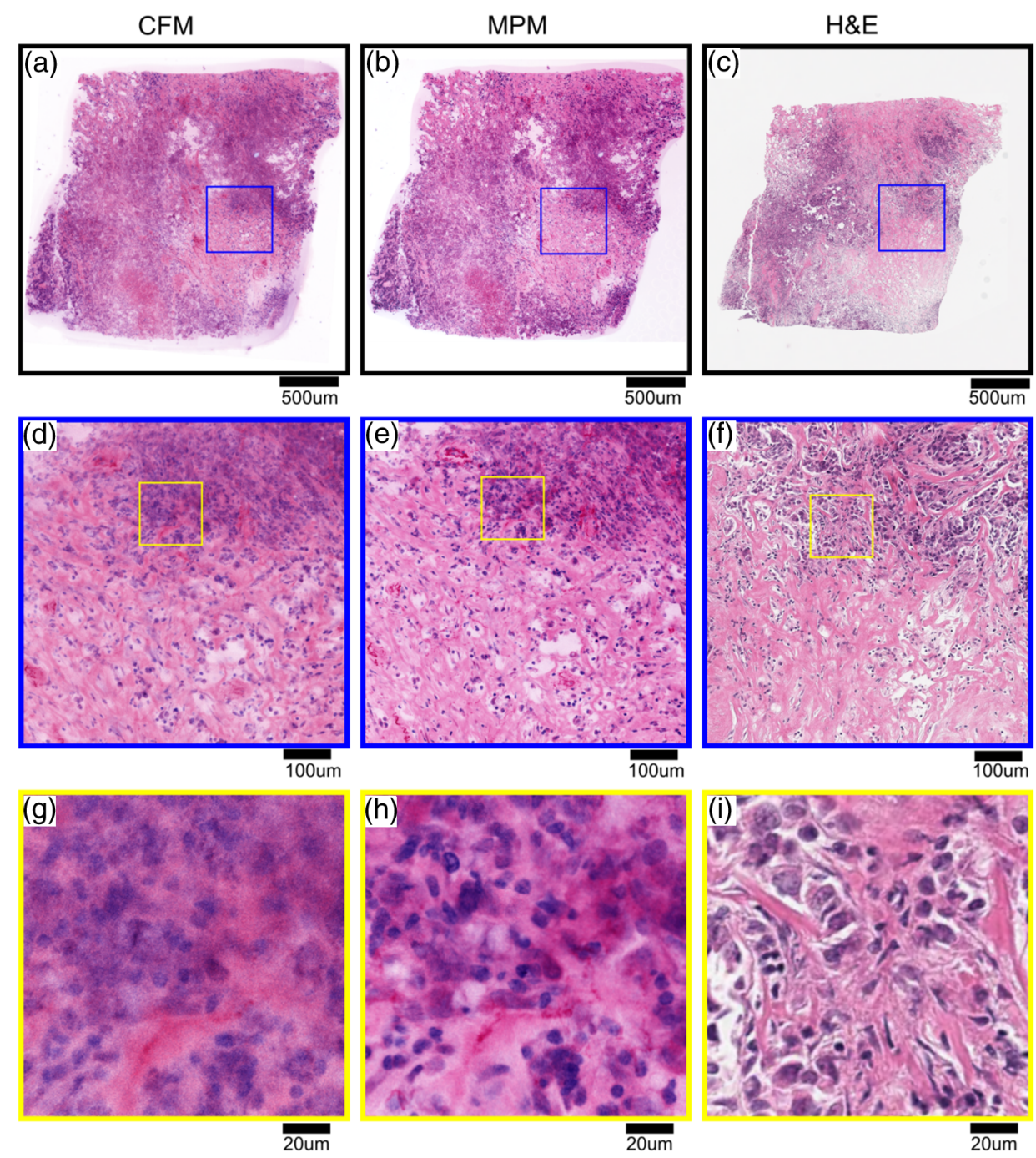

Fig. 4 Comparison of images of an IDC specimen between CFM (20x), MPM (20x), and H\&E histology. (a) CFM image of unfixed breast specimen with areas of viable tumor and zones of geographic necrosis, (b) corresponding MPM image, and (c) corresponding H\&E histology image. (d) Magnified CFM image of carcinoma cells and surrounding tumor stroma, (e) a corresponding MPM image, and (f) corresponding $\mathrm{H} \& \mathrm{E}$ histology image. (h) Higher magnification images of MPM shows image quality comparable to H\&E histology and identify individual nuclei with malignant features. (g) Higher magnification images of CFM shows higher background signal than MPM, but individual nuclei are still identifiable.

$10 \times$ images of the three modalities (Fig. 3, black box and blue box). Further magnified views of CFM and MPM images [Figs. 3(g) and 3(h)] show blurred images, but still allow identification of individual nuclei.

Figure 4 shows $20 \times$ images of breast tissue with invasive ductal carcinoma (IDC) $(2.5 \mathrm{~mm} \times 2.5 \mathrm{~mm})$ from CFM, MPM, and $\mathrm{H} \& \mathrm{E}$ histology. CFM, MPM, and H\&E histology all show cellular areas of viable tumor as well as broad zones of geographic necrosis at low magnification (Fig. 4, black box). Magnified images of a $500 \mu \mathrm{m} \times 500 \mu \mathrm{m}$ area show a similar view of invasive carcinoma and surrounding tumor stroma revealed by the three modalities (Fig. 4, blue box). The higher magnification view of the MPM image [Fig. 4(h)] demonstrates individual nuclei with malignant features, including varying size and shape and increased nuclear/cytoplasmic ratios. The higher magnification view of the CFM image [Fig. 4(g)] shows a noticeable increase of background signal because of the fluorescence from densely aggregated nuclei out of the focal plane. However, most nuclei identifiable in the MPM image are also identifiable in the CFM image.
Figure 5 shows $10 \times$ images of breast tissue with IDC $(2.5 \mathrm{~mm} \times 2.5 \mathrm{~mm})$ from CFM, MPM, and H\&E histology. The features identified at $20 \times$ (Fig. 4) are also seen in the $10 \times$ images from the three modalities (Fig. 5, black box). Magnified images of a $500 \mu \mathrm{m} \times 500 \mu \mathrm{m}$ area show invasive carcinoma and associated tumor stroma (Fig. 5, blue box). A higher magnification view of the MPM image [Fig. 5(h)] shows blurring and a small increase of background signal, but retains sufficient resolution to identify individual nuclei. By contrast, the high magnification view of the CFM image [Fig. 5(g)] shows a significant increase of background signal in addition to blurring and loss of differentiation of individual nuclei.

\subsection{Photobleaching Assessment}

Photobleaching limits the amount of energy that can be applied to any fluorescently labeled specimen as well as the number of times any individual area can be imaged, and it is of particular concern for confocal imaging because all planes within a specimen are 

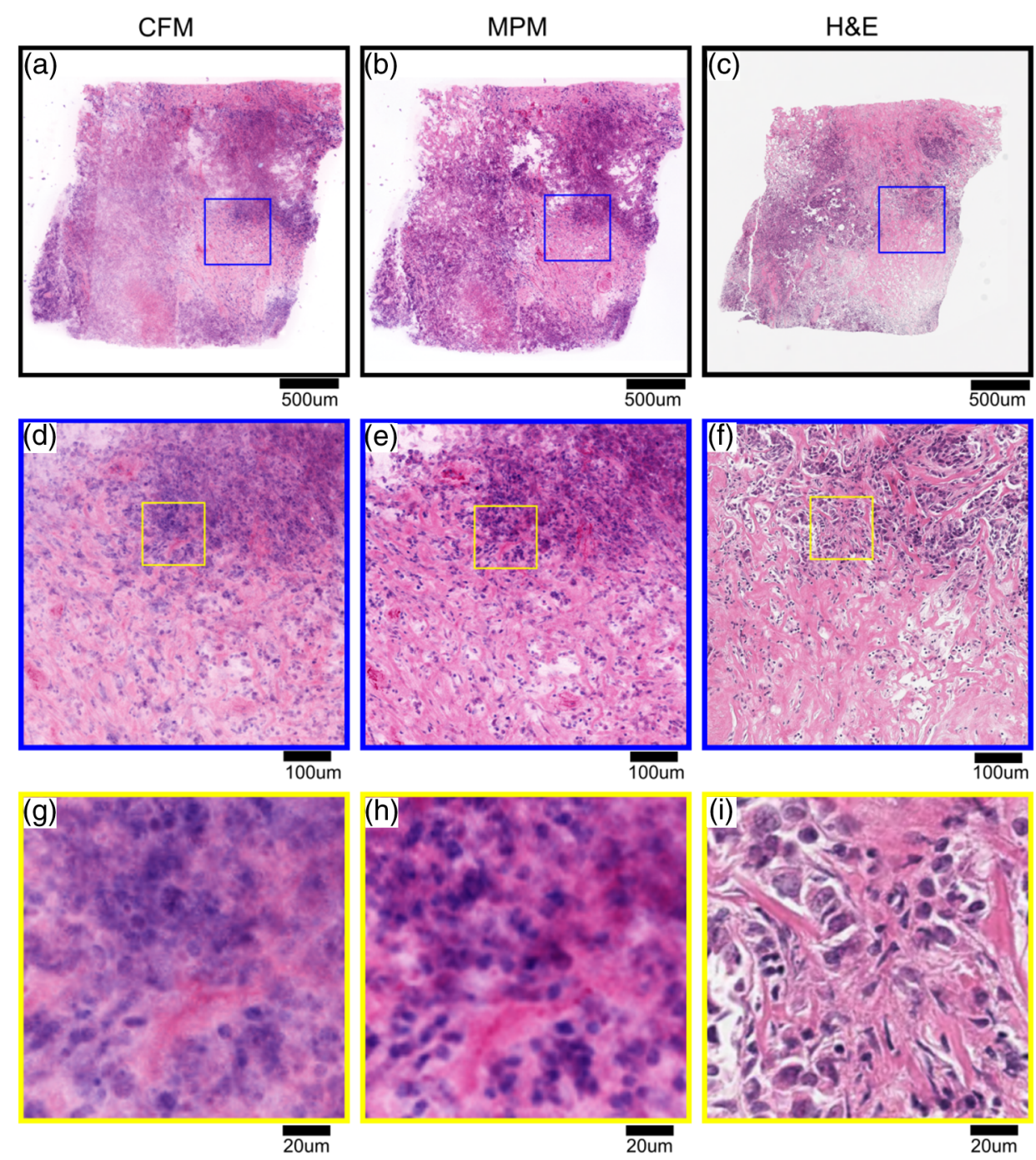

Fig. 5 Comparison of images of an IDC specimen between CFM (10x), MPM (10x), and H\&E histology. (a) CFM image of unfixed breast specimen with viable tumor and geographic necrosis, (b) corresponding MPM image, and (c) corresponding H\&E histology image. (d) Magnified CFM image of carcinoma cells and surrounding tumor stroma, (e) a corresponding MPM image, and (f) corresponding H\&E histology image. (g) Higher magnification image of CFM shows significant increase of background signal, with resultant difficulty in resolving cellular detail. (h) Higher magnification images of MPM show a blurrier image than 20x, but individual nuclei are identifiable. The histology images are identical with those in Fig. 4.

bleached during imaging. To enable intraoperative imaging of surgical specimens, it is important that the staining protocol enables repeatable imaging without substantial photobleaching. Photobleaching effects were assessed for both CFM and MPM using $20 \times$ objectives. One hundred frames were sequentially acquired with the imaging protocol detailed in Sec. 2. Figure 6 shows the normalized fluorescence signals from each contrast agent over 100 frames acquisition. After 100 frames of exposure, the signal magnitudes decayed by $8 \%$ for PI, $11 \%$ for EY in CFM, and $10 \%$ for PI, $7 \%$ for $\mathrm{EY}$ in MPM. Because the effects were small for 100 consecutive frames, we conclude that photobleaching is not a significant limitation for either modality using the staining protocol presented.

\subsection{Imaging Results (Depth)}

The degradation of signal-to-background ratio with increasing image depth in breast specimens was qualitatively evaluated for CFM and MPM using both the $20 \times$ and $10 \times$ objectives. In order to mitigate the effect of attenuation, PMT gains were adjusted to the optimal value for each depth. The nuclear channels are used for evaluation in order to clearly see the degradation in image quality of individual nuclei. Areas with uniform density of nuclei axially were selected to evaluate the effect of imaging depth.

Figure 7 shows CFM and MPM images of normal breast tissue and IDC taken with the $20 \times$ objective at different depths from the surface. Comparing the images of normal and IDC, significant degradation of signal-to-background ratio is seen in the images of IDC in depth [Figs. 7(k), 7(1), 7(o), and 7(p)]. Comparing CFM and MPM, degradation of image quality is more evident in CFM images than MPM images [Figs. 7(d) and $7(\mathrm{~h})$ or $7(\mathrm{k})$, and $7(\mathrm{o})]$. Figure 8 shows the $10 \times$ images of normal breast tissue and IDC specimens at different depths from CFM and MPM. Similar to $20 \times$ images, the degradation of signal-tobackground ratio is significant in IDC images. For both CFM and MPM, individual nuclei are hard to identify in the images 


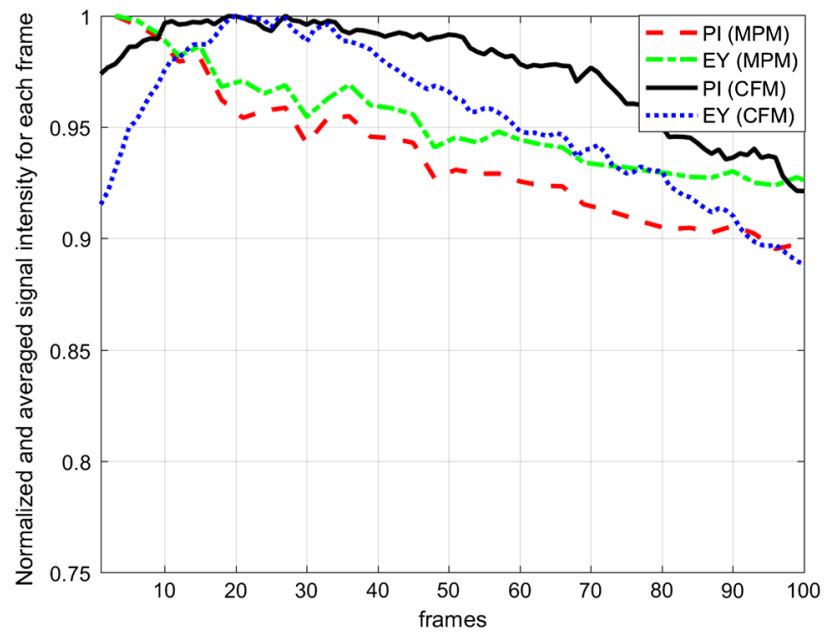

Fig. 6 Photobleaching assessment. The decays of fluorescent signal intensity at the same position were measured in CFM and MPM using $20 \times$ objective until 100 frames of exposure. The maximum signal decay was $11 \%$ at most after 100 frames of exposure. EY had a small but reproducible increase in fluorescent emission after exposure to $405 \mathrm{~nm}$ illumination, possibly due to photochemical reactions in fresh tissue.

at $25 \mu \mathrm{m}$ depth in IDC images. Comparing Figs. 7 and 8, the $20 \times$ images have better image quality with increasing depth than $10 \times$.

\section{Discussion}

In this study, we directly compare CFM, MPM, and paraffinembedded H\&E histology using unfixed human breast tissue, which is representative of what would be encountered in intraoperative histopathological evaluation during BCT. To improve clinical acceptance by pathologists trained on H\&E histopathology, we introduced an imaging protocol using dual channel detection, two contrast agents with rapid penetration into unfixed human tissue (PI and EY), and VH\&E rendering of fluorescent data using the VTM algorithm. Although originally used for MPM imaging, the staining protocol was highly effective for CFM when excited at $405 \mathrm{~nm}$. To evaluate the suitability of CFM and MPM for breast histopathological assessment, images of unfixed breast tissue specimens were acquired using both modalities and compared with corresponding H\&E histology. Photobleaching effects were measured, and the staining protocol was shown to be robust against photobleaching using both MPM and CFM. The degradation of signal-to-background ratio with depth was assessed for both CFM and MPM, and subsurface tissue imaging capability on various tissue types was demonstrated. Although higher magnification objectives were investigated, it was especially important to assess the performance of $10 \times$ magnification objectives, because a large field of view is important for rapid assessment of large tissue specimens in live panning mode.

\subsection{Comparison Between CFM and MPM for High and Low Magnification Imaging}

In typical histopathology workflows, the evaluation of large surgical specimens usually requires both low magnification imaging for assessing broad architectural features and locating diagnostically relevant regions, and higher magnification imaging for analyzing foci of pathologically relevant changes identified at lower magnification. While mosaic mode imaging can be performed using a single magnification, this approach is not needed for intraoperative surgical margin assessment because only the margin of the specimen, rather than the entire face needs to be imaged. Therefore, 10× magnification objectives which would be used for live panning mode imaging were also evaluated for each modality, for both normal and cancerous specimens.

The comparison of the surface images of normal breast tissue from $20 \times$ CFM, 20× MPM, and paraffin-embedded H\&E histology indicates that both CFM and MPM can provide sufficient resolution and axial sectioning to identify individual nuclei in unfixed breast tissues, analogously to formalin-fixed paraffinembedded histology (Fig. 2). Low magnification CFM and MPM imaging of normal breast tissue using $10 \times$ objectives shows that $10 \times$ images were sufficient to allow identification of individual nuclei, although the images have blurring compared with $20 \times$ images because of larger lateral sampling interval (Fig. 3).

High-magnification CFM and MPM imaging of IDC breast tissue using $20 \times$ objectives shows sufficient resolution and axial sectioning to resolve individual nuclei in nuclei-dense malignant tissue (Fig. 4). Although individual nuclei are resolved, the $20 \times$ CFM image of IDC shows a noticeable increase of background signal compared with the $20 \times$ MPM image. Because the axial $20 \%$ to $80 \%$ sectioning of both $20 \times$ CFM and $20 \times$ MPM are comparable, the difference in background signal is due to fluorescence originating significantly out of plane that is incompletely rejected. The high density of nuclei in an invasive carcinoma specimen generates strong fluorescent signal above and below the image plane for CFM, and while the confocal pinhole rejects most out of plane light, in highly fluorescent samples, substantial background signal is still present. This effect is particularly pronounced in Fig. 8 where features at $25 \mu \mathrm{m}$ are directly visible in the underlying depths at 50 and $75 \mu \mathrm{m}$. In contrast, the majority of fluorophore excitation occurs only in the focal plane for MPM, resulting in a noticeably lower background signal and consequently better imaging of nuclei-dense invasive cancer.

In spite of blurring from the coarser lateral sampling and thicker axial sectioning of the $10 \times$ objective, low magnification $10 \times$ MPM imaging of IDC demonstrates that individual cellular features including individual nuclei are readily identifiable over a wider field of view (Fig. 5). Conversely, the 10× CFM image of IDC shows a substantial reduction in image quality, and most individual nuclei are poorly distinguished from the background (Fig. 5). As indicated by the $10 \times$ CFM images of normal tissue specimens, $10 \times \mathrm{CFM}$ is able to clearly visualize isolated individual nuclei in less densely packed specimens in spite of the large optical section thickness. Therefore, the reduction in image quality seen in the IDC image at $10 \times$ is likely due to stronger optical scattering in IDC tissue, the weaker axial sectioning and incomplete rejection of intense out of plane fluorescence from aggregated nuclei. As discussed in Sec. 2.4, the 10× CFM system has an axial section thickness greater than the size of typical cells. Therefore, multiple axially separated nuclei may contribute concurrently to a single voxel, resulting in decreased contrast.

The reduction in signal to background ratio observed in the $10 \times$ CFM images of IDC could be reduced by using higher NA objectives. However, lower magnification objectives are frequently used to survey large areas or assess broad architectural 


\section{Normal Specimen}

(a) :CFM surface

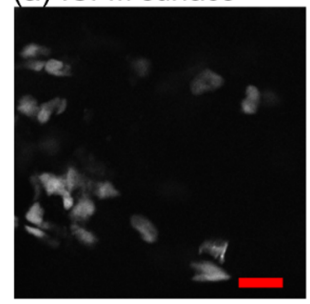

(b) :CFM $+25 \mu \mathrm{m}$

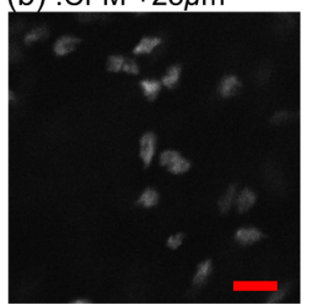

(c) $: \mathrm{CFM}+50 \mu \mathrm{m}$

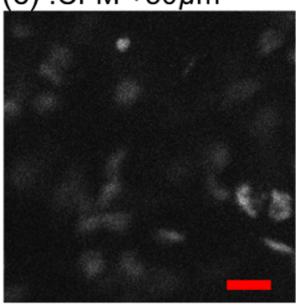

(d) :CFM $+75 \mu \mathrm{m}$

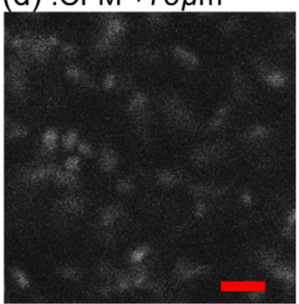

(e) :MPM surface

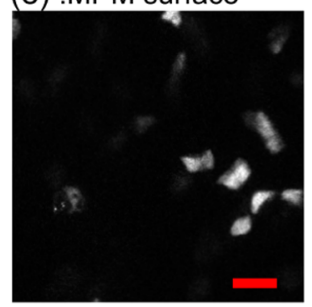

(f) :MPM $+25 \mu \mathrm{m}$

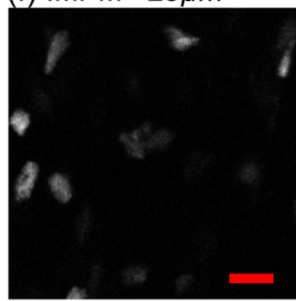

(g) :MPM $+50 \mu \mathrm{m}$

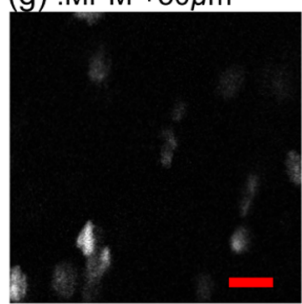

(h) :MPM $+75 \mu \mathrm{m}$

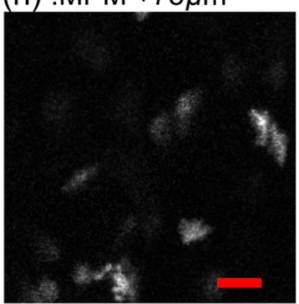

\section{IDC Specimen}
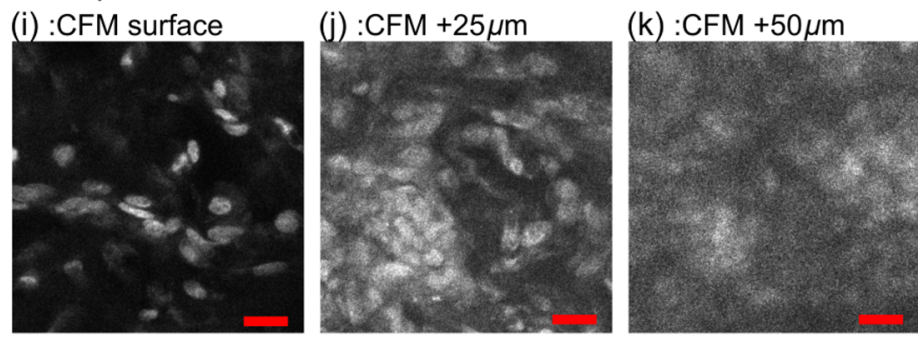

(l) $: \mathrm{CFM}+75 \mu \mathrm{m}$

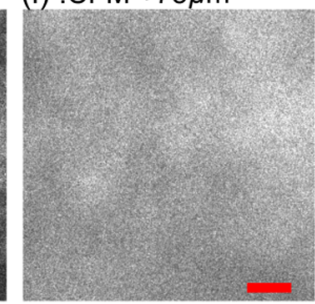

(m) :MPM surface
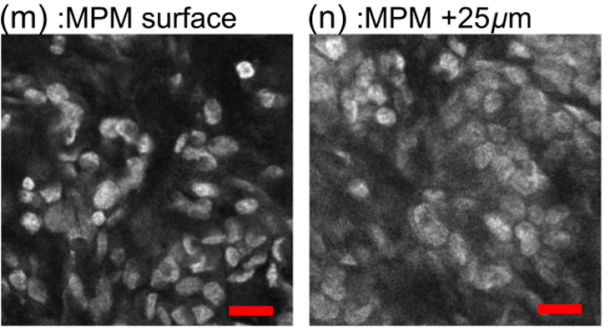

(o) :MPM $+50 \mu \mathrm{m}$

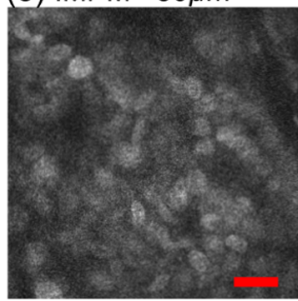

(p) :MPM $+75 \mu \mathrm{m}$

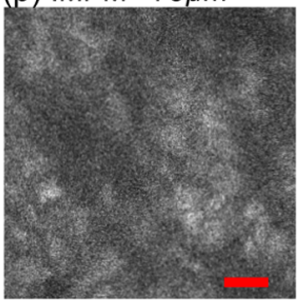

Fig. 7 Nuclear channels (PI) of 20x CFM images of normal breast tissue at different depths [(a) surface, (b) $+25 \mu \mathrm{m}$, (c) $+50 \mu \mathrm{m}$, and (d) $+75 \mu \mathrm{m}$ ], and corresponding 20x MPM images [(e) surface, (f) $+25 \mu \mathrm{m}$, (g) $+50 \mu \mathrm{m}$, and (h) $75 \mu \mathrm{m}$ ]. 20x CFM images of the IDC specimen at different depths [(i) surface, (j) $+25 \mu \mathrm{m}$, (k) $+50 \mu \mathrm{m}$, and (l) $+75 \mu \mathrm{m}]$, and corresponding 20x MPM images $[(\mathrm{m})$ surface, (n) $+25 \mu \mathrm{m}$, (o) $+50 \mu \mathrm{m}$, and (p) $+75 \mu \mathrm{m}$ ]. Degradation of signal-to-background ratio is more significant in the nuclei-rich invasive specimen than in the normal specimen. CFM images show faster degradation of signal-to-background ratio with depth. (scale bar: $20 \mu \mathrm{m}$ )

features, for which identification of individual subcellular features is not required. In this case, the use of a 0.3-NA objective may be sufficient if a higher magnification objective is used to evaluate small areas of pathology. Conversely, the relatively good image quality achieved by 0.3 NA confocal imaging suggests that even lower magnification (e.g., 5×) MPM imaging may be suitable for evaluating some pathologies over very wide fields of view.

\subsection{Imaging Speed}

The data presented in this paper use mosaic mode images that are stitched together after acquisition to provide a comprehensive image of a specimen for comparison to histology slides. Mosaicking is slow, but in actual clinical applications such as
$\mathrm{BCT}$, the specimen is transected or breadloafed and imaging is only required at the margin of the specimen, greatly reducing the area that must be imaged. Therefore user-directed imaging, panning to areas of interest, is likely the preferred workflow because this avoids imaging areas that are irrelevant for clinical evaluation. The finding that both $10 \times$ and $20 \times$ objectives provide high quality $\mathrm{VH} \& \mathrm{E}$ rendering is therefore important because it enables the use of multiple magnifications during user-directed imaging.

In this study, spectrally separated dual channel detectors and two contrast agents were used to improve readability of images by generating VH\&E contrast using PI and EY excited by a single illumination laser. The use of spectrally separated fluorophores that can be efficiently excited by a single laser wavelength is important because it enables higher speed 
Normal Specimen

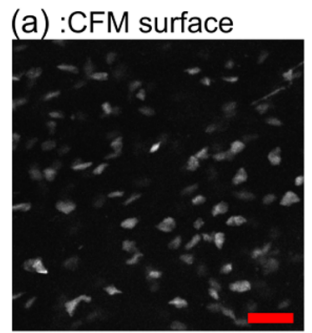

(b) :CFM $+25 \mu \mathrm{m}$

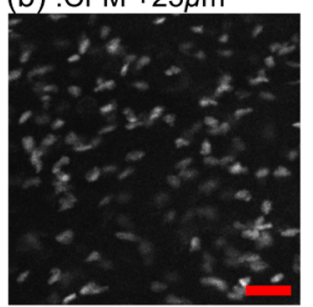

(e) :MPM surface

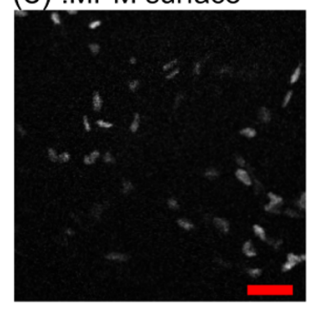

(f) :MPM $+25 \mu \mathrm{m}$
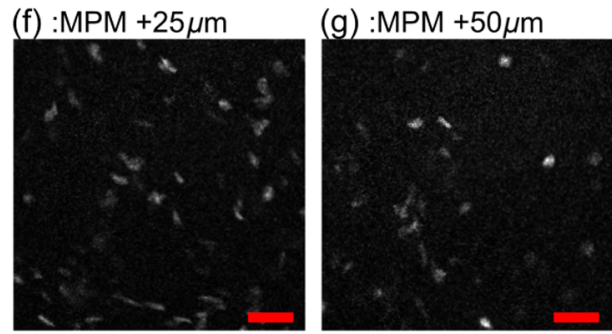

(c) :CFM $+50 \mu \mathrm{m}$

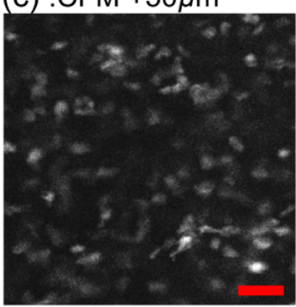

(d) :CFM $+75 \mu \mathrm{m}$

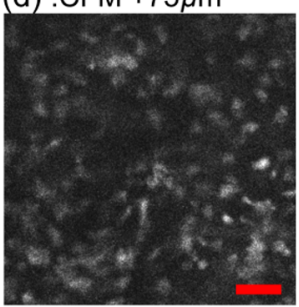

(h) :MPM $+75 \mu \mathrm{m}$

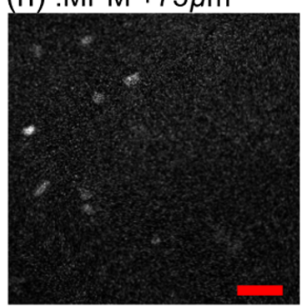

IDC Specimen

(i) :CFM surface

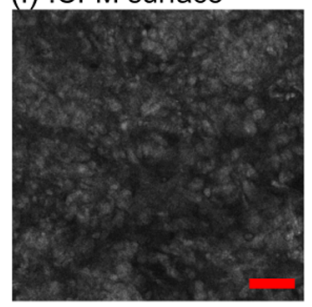

(j) :CFM $+25 \mu \mathrm{m}$

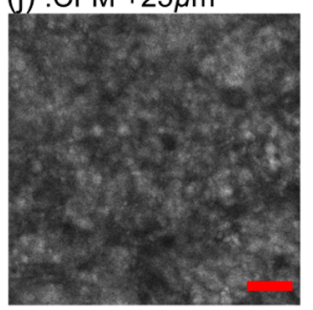

(k) :CFM $+50 \mu \mathrm{m}$

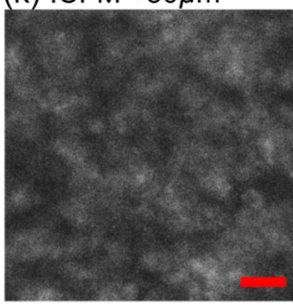

(l) $: \mathrm{CFM}+75 \mu \mathrm{m}$

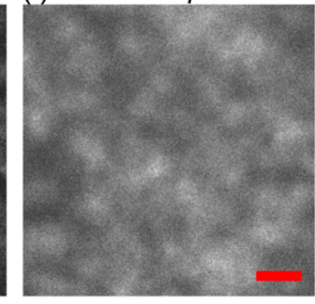

(m) :MPM surface

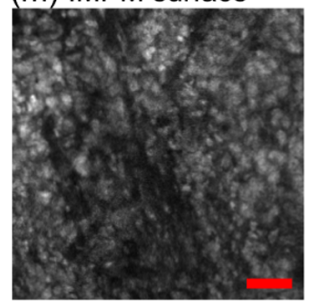

(n) :MPM $+25 \mu \mathrm{m}$

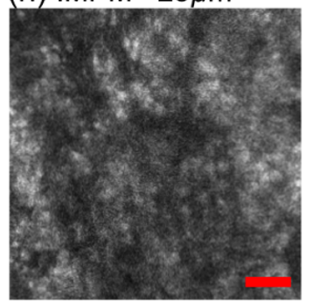

(o) :MPM $+50 \mu \mathrm{m}$

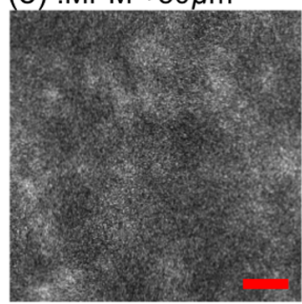

(p) :MPM $+75 \mu \mathrm{m}$

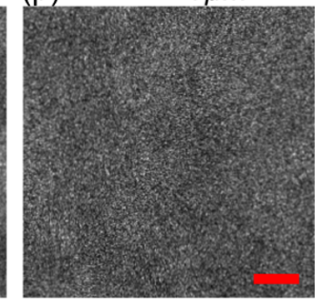

Fig. 8 Nuclear channels $(\mathrm{PI})$ of 10x CFM images of the normal breast tissue at different depths [(a) surface, (b) $+25 \mu \mathrm{m},(\mathrm{c})+50 \mu \mathrm{m}$, and (d) $+75 \mu \mathrm{m}]$, and corresponding 10× MPM images [(e) surface, (f) $+25 \mu \mathrm{m}$, (g) $+50 \mu \mathrm{m}$, and (h) $75 \mu \mathrm{m}]$. 10x CFM images of the IDC specimen at different depths [(i) surface, (j) $+25 \mu \mathrm{m},(\mathrm{k})+50 \mu \mathrm{m}$, and (l) $+75 \mu \mathrm{m}$ ], and corresponding 10x MPM images [(m) surface, (n) $+25 \mu \mathrm{m}$, (o) $+50 \mu \mathrm{m}$, and (p) $+75 \mu \mathrm{m}]$. Degradation of signal-to-background ratio is more significant in the nuclei-rich invasive specimen than in the normal specimen. (scale bar: $40 \mu \mathrm{m}$ )

imaging than multiplexing excitation wavelengths. In our MPM system, the use of resonant galvo scanning enabled video-rate imaging at 16 frames per second, while GPU processing enabled latency free $\mathrm{VH} \& \mathrm{E}$ rendering with the VTM algorithm. In the CFM system, the per pixel dwell time was 50-times-longer than the MPM system because of the nonresonant galvo scanning. Imaging speeds could be improved by using resonant scanning for CFM, as was used for MPM, or possibly by parallel pinhole scanning, although we note that techniques such as spinning disk may incur substantial crosstalk between pinholes when imaging highly scattering specimens such as human tissue. ${ }^{33}$ Higher imaging speed in CFM is ultimately limited by fluorophore saturation, which occurs when illumination power is sufficiently large such that few unexcited fluorophores are available within a focal volume. However, the MPM system, which had a smaller focal volume than either CFM configurations and an inherently less efficient use of pixel time due to the fixed laser pulse rate, did not encounter fluorophore saturation even at much higher illumination powers than were used in this study, indicating that the density of fluorophores in human tissue stained using the current protocol is sufficient to support extremely high imaging speeds. A further concern is that more rapid scanning might decrease the absolute time required to photobleach a specimen; however, we note that there is negligible photobleaching with our staining protocol even after 100 sequential frames and photobleaching would scale as the number of frames, irrespective of the frame rate.

\subsection{Imaging in Depth}

As shown in Figs. 7 and 8, the signal-to-background ratio of CFM and MPM degrades with increasing depth into specimens. 
MPM has superior signal-to-background ratio with depth compared to CFM because, for CFM, scattering affects both illumination intensity and fluorescence background from outside of the imaging plane. In MPM, scattering affects illumination intensity, but scattering of fluorescence from the two photon absorption does not significantly contribute to the background because excitation is localized to the imaging plane. ${ }^{34}$ In addition, the wavelength of illumination of MPM $(780 \mathrm{~nm}$ in this study) is approximately twice as long as that of CFM (405 nm in this study), greatly reducing the scattering coefficient of excitation light for MPM. Finally, specimen induced spherical aberration likely degrades image quality to some extent for the high NA objectives. This effect could be reduced by using a water immersion objective for $20 \times \mathrm{CFM}$; however, as evidenced by the large difference in imaging depth between normal and IDC specimens, the primary limitation on imaging depth appears to be scattering of the 405-nm illumination and out of plane fluorescence rather than spherical aberration.

The imaging results at different depths indicate that tissue characteristics significantly affect the imaging performance with depth, independent of the objective and imaging modality. As shown in Figs. 7 and 8, the degradation of image quality in IDC is much more pronounced for both CFM and MPM than in normal breast tissue because IDC is more scattering and has a high density of fluorescent nuclei that contribute to the background signal. Consequently, imaging depth in IDC is more limited for both modalities as compared to normal tissue, although this limitation is more pronounced for CFM than MPM.

Finally, we note that although both MPM and CFM have limited maximum imaging depths due to the high scattering coefficients of breast tissue, it is uncommon in clinical practice to examine serial sections of breast surgical margins. As noted, specimens are transected or breadloafed into multiple slices on the order of several $\mathrm{mm}$ to $1 \mathrm{~cm}$ thickness to represent the surgical margins, and that are then imaged on or near the transected surface plane. Therefore, the limited image depths in specimens would not be a limitation in current clinical workflows.

\section{Conclusion}

We report a direct comparison between CFM and MPM with dual agent staining and rendering of VH\&E images using the VTM algorithm for rapid histopathological evaluation of unfixed human breast tissue. We demonstrate that CFM can image the surface of unfixed breast tissue with comparable quality to MPM. Using the dual staining protocol presented and a light absorption VTM model, both modalities can generate images to correspond closely to H\&E histology. Photobleaching effects are shown to be negligible even after 100 sequential images of the same location. Wide area mosaicking of large specimens enables comprehensive comparison between CFM, MPM, and H\&E. The images of the surfaces of the specimens demonstrate that $20 \times$ CFM and $20 \times$ MPM have comparable imaging performance for individual nuclei in both normal breast tissue and IDC. Both $10 \times$ CFM and 10× MPM show degraded image quality as compared with $20 \times$ due to reduced lateral resolution and axial sectioning, but still enable visualization of individual nuclei in normal breast tissue. The 10× CFM images showed a significant increase in background signal and loss of individual nuclei identification when imaging IDC because of the reduced optical sectioning and increased contribution of out of focal plane signal, although this effect was partially attributable to the lower objective NA.

The main limitations of this comparison are the modest difference in numerical apertures between the CFM system and MPM system and difference of immersion media. However, even at slightly lower NA, CFM demonstrated excellent correspondence with both MPM and paraffin-embedded histology, and in normal workflows using two objectives, higher NA and immersion media may be unnecessary. These results suggest that CFM may be a promising and cost-effective alternative to MPM for BCT.

\section{Disclosures}

T.Y. was employed by Canon Inc. during the time this research was performed, but the authors declare no competing interest.

\section{Acknowledgments}

We gratefully acknowledge helpful advice from Dr. Ben Potsaid. This study was supported in part by the National Institutes of Health (Grant Nos. R01-CA178636-02, R01-CA075289-18, and F32-CA183400-03) and Air Force Office of Scientific Research AFOSR contracts (Grant Nos. FA9550-12-1-0551 and FA9550-15-1-0473).

\section{References}

1. F. J. Fleming et al., "Intraoperative margin assessment and re-excision rate in breast conserving surgery," Eur. J. Surg. Oncol. 30(3), 233-237 (2004).

2. A. Kobbermann et al., "Impact of routine cavity shave margins on breast cancer re-excision rates," Ann. Surg. Oncol. 18(5), 1349-1355 (2011).

3. L. E. McCahill et al., "Variability in reexcision following breast conservation surgery," J. Am. Med. Assoc. 307(5), 467-475 (2012).

4. P. J. van Diest et al., "Reliability of intraoperative frozen section and imprint cytological investigation of sentinel lymph nodes in breast cancer," Histopathology 35(1), 14-18 (1999).

5. U. Veronesi et al., "Sentinel-node biopsy to avoid axillary dissection in breast cancer with clinically negative lymph-nodes," Lancet 349(9069), 1864-1867 (1997).

6. S. A. McLaughlin et al., "Influence of frozen-section analysis of sentinel lymph node and lumpectomy margin status on reoperation rates in patients undergoing breast-conservation therapy," J. Am. Coll. Surg. 206(1), 76-82 (2008).

7. T. S. Menes et al., "Touch preparation or frozen section for intraoperative detection of sentinel lymph node metastases from breast cancer," Ann. Surg. Oncol. 10(10), 1166-1170 (2003).

8. R. G. Pleijhuis et al., "Obtaining adequate surgical margins in breastconserving therapy for patients with early-stage breast cancer: current modalities and future directions," Ann. Surg. Oncol. 16(10), 2717-2730 (2009).

9. T. P. Olson et al., "Frozen section analysis for intraoperative margin assessment during breast-conserving surgery results in low rates of re-excision and local recurrence," Ann. Surg. Oncol. 14(10), 29532960 (2007).

10. O. Riedl et al., "Intraoperative frozen section analysis for breastconserving therapy in 1016 patients with breast cancer," Eur. J. Surg. Oncol. 35(3), 264-270 (2009).

11. S. L. Blair et al., "Attaining negative margins in breast-conservation operations: is there a consensus among breast surgeons?," J. Am. Coll. Surg. 209(5), 608-613 (2009).

12. M. Rajadhyaksha et al., "In vivo confocal scanning laser microscopy of human skin II: advances in instrumentation and comparison with histology," J. Invest. Dermatol. 113(3), 293-303 (1999).

13. M. Rajadhyaksha, R. R. Anderson, and R. H. Webb, "Video-rate confocal scanning laser microscope for imaging human tissues in vivo," Appl. Opt. 38(10), 2105-2115 (1999).

14. M. Rajadhyaksha et al., "Confocal examination of nonmelanoma cancers in thick skin excisions to potentially guide mohs micrographic 
surgery without frozen histopathology," J. Invest. Dermatol. 117(5), 1137-1143 (2001).

15. G. Pellacani, A. M. Cesinaro, and S. Seidenari, "Reflectance-mode confocal microscopy of pigmented skin lesions-improvement in melanoma diagnostic specificity," J. Am. Acad. Dermatol. 53(6), 979-985 (2005).

16. D. S. Gareau et al., "Fluorescence confocal mosaicing microscopy of basal cell carcinomas to potentially guide mohs surgery," in OSA Technical Digest (CD), Biomedical Optics BTuB3 (2008).

17. D. S. Gareau et al., "Confocal mosaicing microscopy in Mohs skin excisions: feasibility of rapid surgical pathology," J. Biomed. Opt. 13(5), 054001 (2008).

18. D. S. Gareau et al., "Sensitivity and specificity for detecting basal cell carcinomas in Mohs excisions with confocal fluorescence mosaicing microscopy," J. Biomed. Opt. 14(3), 034012 (2009).

19. J. K. Karen et al., "Detection of basal cell carcinomas in Mohs excisions with fluorescence confocal mosaicing microscopy," Br. J. Dermatol. 160(6), 1242-1250 (2009).

20. J. Bini et al., "Confocal mosaicing microscopy of human skin ex vivo: spectral analysis for digital staining to simulate histology-like appearance," J. Biomed. Opt. 16(7), 076008 (2011).

21. C. Longo et al., "Evaluating ex vivo fluorescence confocal microscopy images of basal cell carcinomas in Mohs excised tissue," $\mathrm{Br}$. $J$. Dermatol. 171(3), 561-570 (2014).

22. F. Helmchen and W. Denk, "Deep tissue two-photon microscopy," Nat. Methods 2(12), 932-940 (2005).

23. I. Pavlova et al., "Multiphoton microscopy and microspectroscopy for diagnostics of inflammatory and neoplastic lung," J. Biomed. Opt. 17(3), 036014 (2012).

24. Y. K. Tao et al., "Assessment of breast pathologies using nonlinear microscopy," Proc. Natl. Acad. Sci. U. S. A. 111(43), 15304-15309 (2014).

25. M. Wang et al., "High-resolution rapid diagnostic imaging of whole prostate biopsies using video-rate fluorescence structured illumination microscopy," Cancer Res. 75(19), 4032-4041 (2015).
26. E. F. Brachtel et al., "Spectrally encoded confocal microscopy for diagnosing breast cancer in excision and margin specimens," Lab. Invest. 96(4), 459-467 (2016).

27. S. A. Boppart et al., "Optical coherence tomography: feasibility for basic research and image-guided surgery of breast cancer," Breast Cancer Res. Treat. 84(2), 85-97 (2004).

28. J. G. Fujimoto et al., "Optical biopsy and imaging using optical coherence tomography," Nat. Med. 1(9), 970-972 (1995).

29. M. Ragazzi et al., "Fluorescence confocal microscopy for pathologists," Mod. Pathol. 27(3), 460-471 (2014).

30. J. L. Dobbs et al., "Feasibility of confocal fluorescence microscopy for real-time evaluation of neoplasia in fresh human breast tissue," J. Biomed. Opt. 18(10), 106016 (2013).

31. M. G. Giacomelli et al., "Virtual hematoxylin and eosin transillumination microscopy using epi-fluorescence imaging," PLOS ONE 11(8), e0159337 (2016).

32. E. H. K. Stelzer et al., "Nonlinear absorption extends confocal fluorescence microscopy into the ultra-violet regime and confines the illumination volume," Opt. Commun. 104(4), 223-228 (1994).

33. R. Wolleschensky, B. Zimmermann, and M. Kempe, "High-speed confocal fluorescence imaging with a novel line scanning microscope," J. Biomed. Opt. 11(6), 064011 (2006).

34. V. E. Centonze and J. G. White, "Multiphoton excitation provides optical sections from deeper within scattering specimens than confocal imaging," Biophys. J. 75(4), 2015-2024 (1998).

Tadayuki Yoshitake is a PhD candidate in the Department of Electrical Engineering and Computer Science and the Research Laboratory of Electronics at the Massachusetts Institute of Technology. He received his $B S$ and $M S$ degrees in electrical engineering from Kyoto University (Japan) in 2006 and 2008, respectively. After several years of industry experience, his current research interests include optical sectioning microscopy and its application to pathology.

Biographies for the other authors are not available. 